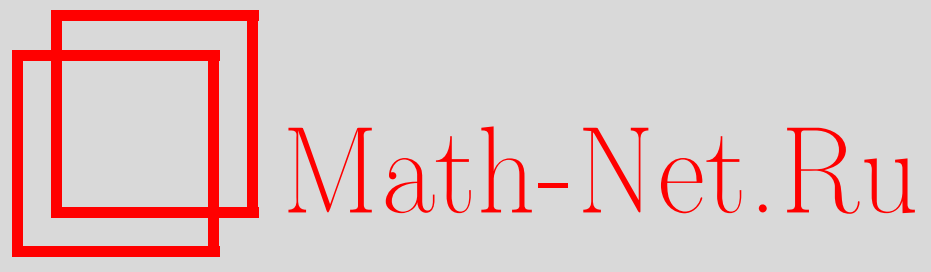

В. Л. Камынин, Об обратной задаче определения старшего коэффициента в параболическом уравнении, Maтем. заметки, 2008, том 84, выпуск 1, 48-58

DOI: https://doi.org/10.4213/mzm4134

Использование Общероссийского математического портала Math-Net.Ru подразумевает, что вы прочитали и согласны с пользовательским соглашением http://www . mathnet.ru/rus/agreement

Параметры загрузки:

IP : 52.87 .193 .239

26 апреля 2023 г., 16:53:12

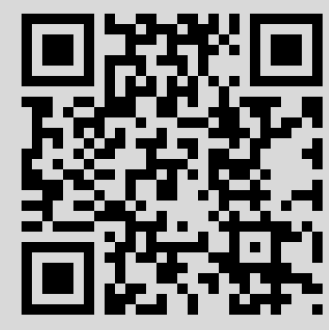


Том 84 выпуск 1 июль 2008

УДК 517.956

\section{Об обратной задаче определения старшего коэффициента в параболическом уравнении}

\section{В. Л. Камынин}

Изучается вопрос об однозначной разрешимости обратной задачи определения старшего коэффициента для параболического уравнения на плоскости с коэффициентами, зависящими и от временной и от пространственной переменной при условии интегрального переопределения по времени. Получены условия, достаточные для однозначной разрешимости рассмотренной обратной задачи. Приведены нетривиальные примеры задач, для которых такие условия выполняются. Показано, что наложенные условия заведомо выполнены, если либо интервал времени достаточно велик, либо пространственный отрезок, на котором рассматривается задача, достаточно мал.

Библиография: 9 названий.

1. Введение. В работе изучаются вопросы существования и единственности решения $\{u(t, x), a(x)\}$ нелинейной обратной задачи в $Q_{T} \equiv[0, T] \times[0, l]$ для уравнения

$$
\rho(t, x) u_{t}-a(x) u_{x x}+b(x) u_{x}+d(t, x) u=f(t, x),
$$

с начальным условием

$$
u(0, x)=0, \quad x \in[0, l],
$$

граничными условиями

$$
u(t, 0)=u(t, l)=0, \quad t \in[0, T],
$$

при дополнительном условии

$$
\int_{0}^{T} u(t, x) \chi(t) d t=\varphi(x), \quad x \in[0, l] .
$$

Различным обратным задачам для параболических уравнений посвящена обширная литература, однако работ, где к числу неизвестных относится коэффициент при старшей производной, известно немного.

Прежде всего, это работа [1], где рассматривалась обратная задача определения старшего коэффициента в многомерном параболическом уравнении с переопределением (1.4), а также и с финальным переопределением вида

$$
u(T, x)=\psi(x),
$$

но при условии, что коэффициенты уравнения не зависят от $t$.

Работа выполнена при поддержке Российского фонда фундаментальных исследований (грант № 06-01-00401).

(C) В. Л. КАмынин, 2008 
Отметим также работы [2], где получена разрешимость обратной задачи с финальным переопределением (1.5) для параболического уравнения с гладкими коэффициентами [3], [4], где получена единственность решения обратной задачи с переопределением (1.5) для уравнения с гладкими коэффициентами, и [5], где изучалась обратная задача с условием переопределения во внутренней точке $x_{0} \in(0, l)$.

Пространства $L_{2}([0, l]), L_{2}\left(Q_{T}\right), L_{\infty}([0, l]), W_{2}^{1}([0, l]), W_{2}^{1}\left(Q_{T}\right), W_{2}^{2}([0, l]), W_{2}^{1,2}\left(Q_{T}\right)$, $W_{\infty}^{2}([0, l]), C^{1, \gamma}\left(Q_{T}\right)$ с соответствующими нормами будем понимать в общепринятом смысле (см., например, [6], [7]). Через $W_{2,0}^{1,2}\left(Q_{T}\right)$ (соответственно $\stackrel{\circ}{W} \underset{2}{1}\left(Q_{T}\right), \stackrel{\circ}{W_{2}^{1}}([0, l])$, $\left.W_{2,0}^{2}([0, l])\right)$ будем обозначать подпространство функций из $W_{2}^{1,2}\left(Q_{T}\right)$ (соответственно из $\left.W_{2}^{1}\left(Q_{T}\right), W_{2}^{1}([0, l]), W_{2}^{2}([0, l])\right)$, равных нулю при $x=0, l$.

Будем использовать известное арифметическое неравенство

$$
|a b| \leqslant \frac{\varepsilon}{2} a^{2}+\frac{1}{2 \varepsilon} b^{2}, \quad \varepsilon>0, \quad a, b \in \mathbb{R}^{1} .
$$

Заметим, что для функций $z(x) \in \stackrel{\circ}{W_{2}^{1}}([0, l])$ справедливо неравенство ПуанкареСтеклова, которое для $n=1$ имеет вид

$$
\|z\|_{L_{2}([0, l])}^{2} \leqslant \frac{l^{2}}{2}\left\|z_{x}\right\|_{L_{2}([0, l])}^{2} .
$$

Для функций $z(x) \in W_{2,0}^{2}([0, l])$ имеем

$$
\int_{0}^{x} z_{x}^{2} d x=-\int_{0}^{x} z z_{x x} d x \leqslant\|z\|_{L_{2}([0, l])} \cdot\left\|z_{x x}\right\|_{L_{2}([0, l])},
$$

откуда, применяя (1.7), выводим неравенство

$$
\left\|z_{x}\right\|_{L_{2}([0, l])}^{2} \leqslant \frac{l^{2}}{2}\left\|z_{x x}\right\|_{L_{2}([0, l])}^{2} .
$$

Далее, для $z(x) \in \stackrel{\circ}{W_{2}^{1}}([0, l])$ имеем $z(x)=\int_{0}^{x} z_{y}(y) d y$, откуда следует, что

$$
\|z\|_{L_{\infty}([0, l])} \leqslant l^{1 / 2}\left\|z_{x}\right\|_{L_{2}([0, l])} .
$$

Наконец, используя (1.9), легко получить неравенство

$$
\int_{0}^{T}\|v(t, \cdot)\|_{L_{\infty}([0, l])} d t \leqslant(l T)^{1 / 2}\left\|v_{x}\right\|_{L_{2}\left(Q_{T}\right)}, \quad v(t, x) \in \stackrel{\circ}{W_{2}^{1}}\left(Q_{T}\right) .
$$

Во всех дальнейших рассуждениях мы будем предполагать, что функции, входящие в исходные данные задачи (1.1)-(1.4), измеримы и удовлетворяют следующим условиям:
(A) $0<\rho_{1} \leqslant \rho(t, x) \leqslant \rho_{2}, \quad-\rho^{*} \leqslant \rho_{t}(t, x) \leqslant \rho^{* *}, \quad(t, x) \in Q_{T}$;
(B) $|b(x)| \leqslant K_{b}, \quad x \in[0, l]$;
(C) $-d_{1} \leqslant d(t, x) \leqslant d_{2}, \quad(t, x) \in Q_{T}$;
(D) $0 \leqslant f_{1} \leqslant f(t, x) \leqslant f_{2}, \quad(t, x) \in Q_{T}$; 


$$
\begin{aligned}
\text { (E) } \varphi(x) \in W_{\infty}^{2}([0, l]), \quad \varphi(0)=\varphi(l)=0, \quad \varphi(x) \geqslant 0, \\
\\
-\varphi_{2} \leqslant \varphi^{\prime \prime}(x) \leqslant-\varphi_{1}<0, \quad\left|\varphi^{\prime}(x)\right| \leqslant \varphi^{*}, \quad x \in[0, l] ; \\
\text { (F) } \quad 0 \leqslant \chi_{1} \leqslant \chi(t) \leqslant \chi_{2}, \quad-\chi^{*} \leqslant \chi^{\prime}(t) \leqslant \chi^{* *}, \quad t \in[0, T] .
\end{aligned}
$$

Здесь $\rho_{1}, \rho_{2}, \varphi_{1}, \varphi_{2}, \varphi^{*}, \chi_{2}, f_{2}=$ const $>0 ; \rho^{*}, \rho^{* *}, \chi_{1}, \chi^{*}, \chi^{* *}, f_{1}, K_{b}, d_{1}, d_{2}=$ const $\geqslant 0$.

Обозначим $K_{\rho}=\max \left\{\rho^{*}, \rho^{* *}\right\}, K_{\chi}=\max \left\{\chi^{*}, \chi^{* *}\right\}, K_{d}=\max \left\{d_{1}, d_{2}\right\}$. Положим $F(x, T)=\int_{0}^{T} f(t, x) \chi(t) d t$ и обозначим

$$
F_{1}(T)=\inf _{x \in[0, l]} F(x, T), \quad F_{2}(T)=\sup _{x \in[0, l]} F(x, T) .
$$

Введем еще множество $M_{\delta}=\left\{a(x) \in L_{\infty}([0, l]): a(x) \geqslant \delta=\right.$ const $\left.>0\right\}$.

ОПРЕДЕЛЕНИЕ. Обобщенным решением задачи (1.1)-(1.4) будем называть пару функций $\{u(t, x), a(x)\}, u(t, x) \in W_{2}^{1,2}\left(Q_{T}\right) \cap C^{1, \gamma}\left(Q_{T}\right), \gamma=$ const $\in(0,1), a(x) \in M_{\delta}$, такую, что эта пара удовлетворяет уравнению (1.1) почти всюду в $Q_{T}$ и при этом функция $u(t, x)$ удовлетворяет условиям (1.2)-(1.4).

2. Существование решения обратной задачи (1.1)-(1.4). Выведем операторное уравнение относительно неизвестной функции $a(x)$.

Пусть $a(x) \in M_{\delta}$ произвольна. Тогда соотношения (1.1)-(1.3) представляют собой первую краевую задачу для нахождения функции $u(t, x)$. В силу [7] обобщенное решение $u(t, x) \in W_{2}^{1,2}\left(Q_{T}\right) \cap C^{1, \gamma}\left(Q_{T}\right)$ этой задачи существует и единственно. Умножим (1.1) на $\chi(t)$ и проинтегрируем по $t$ в пределах от 0 до $T$. Учитывая условия (A), (E), (F), а также (1.2)-(1.4), приходим к соотношению

$$
\begin{aligned}
a(x)=\frac{1}{-\varphi^{\prime \prime}(x)}\left[\int_{0}^{T}(\rho(t, x) \chi(t))_{t} u(t, x) d t-\int_{0}^{T} d(t, x) \chi(t) u(t, x) d t\right. & \\
& \left.-b(x) \varphi^{\prime}(x)-\rho(T, x) \chi(T) u(T, x)+F(x, T)\right] .
\end{aligned}
$$

Введем нелинейный оператор $B$ по формуле

$$
\begin{aligned}
B(a)=\frac{1}{-\varphi^{\prime \prime}(x)}\left[\int_{0}^{T}(\rho(t, x) \chi(t))_{t} u(t, x) d t-\int_{0}^{T} d(t, x) \chi(t) u(t, x) d t\right. & \\
& \left.-b(x) \varphi^{\prime}(x)-\rho(T, x) \chi(T) u(T, x)+F(x, T)\right],
\end{aligned}
$$

где $u(t, x)$ - обобщенное решение прямой задачи (1.1)-(1.3) с коэффициентом $a(x)$ в уравнении (1.1). Тогда соотношение (2.1) можно записать в виде операторного уравнения

$$
a=B(a)
$$

ЛЕмма 2.1. Пусть выполнены условия (A)-(F). Тогда, для того чтобы пара $\{u(t, x), a(x)\}$ была обобщенным решением задачи (1.1)-(1.4), необходимо и достаточно, чтобы эта пара удовлетворяла соотношениям (1.1)-(1.3), (2.3).

ДокАЗАТЕЛЬство. Необходимость доказана выше при выводе соотношения (2.3). 
Докажем достаточность. Пусть $\widetilde{a}(x) \in M_{\delta}$ является решением уравнения (2.3). Рассмотрим функцию $\widetilde{u}(t, x)$ как единственное обобщенное решение прямой задачи (1.1)-(1.3) с выбранным коэффициентом $a(x) \equiv \widetilde{a}(x)$ в уравнении (1.1). Положим $\widetilde{\varphi}(x)=\int_{0}^{T} \widetilde{u}(t, x) \chi(t) d t$. Очевидно, $\widetilde{\varphi}(x) \in W_{2,0}^{2}([0, l])$. Повторяя рассуждения, проведенные выше при выводе (2.1) (в этих рассуждениях достаточно, чтобы $\widetilde{\varphi}(x) \in W_{2,0}^{2}([0, l])$, приходим к соотношению

$$
\begin{gathered}
\left(-\widetilde{\varphi}^{\prime \prime}\right) \widetilde{a}(x)=\int_{0}^{T}(\rho(t, x) \chi(t))_{t} \widetilde{u}(t, x) d t-\int_{0}^{T} d(t, x) \chi(t) \widetilde{u}(t, x) d t-b(x) \widetilde{\varphi}^{\prime} \\
-\rho(T, x) \chi(T) \widetilde{u}(T, x)+F(x, T) .
\end{gathered}
$$

С другой стороны, $\widetilde{a}(x)$ является решением уравнения (2.3), поэтому, учитывая определение оператора $B$ в (2.2), находим, что

$$
\begin{gathered}
\left(-\varphi^{\prime \prime}\right) \widetilde{a}(x)=\int_{0}^{T}(\rho(t, x) \chi(t))_{t} \widetilde{u}(t, x) d t-\int_{0}^{T} d(t, x) \chi(t) \widetilde{u}(t, x) d t-b(x) \varphi^{\prime} \\
-\rho(T, x) \chi(T) \widetilde{u}(T, x)+F(x, T) .
\end{gathered}
$$

Вычитая (2.4) из (2.5), получаем, что на отрезке $[0, l]$

$$
(\widetilde{\varphi}-\varphi)^{\prime \prime}=\frac{b(x)}{\widetilde{a}(x)}(\widetilde{\varphi}-\varphi)^{\prime},
$$

откуда с учетом принадлежности функций $\widetilde{\varphi}(x), \varphi(x)$ пространству $W_{2,0}^{2}([0, l])$ вытекает, что $\widetilde{\varphi}(x)=\varphi(x)$ на $[0, l]$, а следовательно, пара $\{\widetilde{u}(t, x), \widetilde{a}(x)\}$ является обобщенным решением обратной задачи (1.1)-(1.4). Лемма 2.1 доказана.

Всюду ниже в дополнение к условиям (A)-(F) будем предполагать выполненным неравенство

$$
F_{1}(T)-K_{b} \varphi^{*}>\frac{3 \sqrt{3} \rho_{2} \varphi_{2}}{\sqrt{2} \rho_{1}}\left(K_{b}^{2}+\frac{K_{d}^{2} l^{2}}{2}\right)^{1 / 2} l .
$$

Положим

$$
a_{1}=\frac{F_{1}(T)-K_{b} \varphi^{*}}{3 \varphi_{2}} .
$$

Заметим, что в силу (2.6) число $a_{1}$ из (2.7) является положительным.

Выведем ряд оценок для решения прямой задачи (1.1)-(1.3) с $a(x) \in M_{a_{1}}$, где $a_{1}$ из (2.7).

Лемма 2.2. Пусть выполнены условия (A)-(F), (2.6) и а $a_{1}$ из (2.7). Тогда для обобщенного решения $u(t, x)$ задачи (1.1)-(1.3) с а $(x) \in M_{a_{1}}$ справедливы оченки

$$
\begin{aligned}
\left\|u_{x}(\tau, \cdot)\right\|_{L_{2}([0, l])}^{2} & \leqslant \frac{3 \rho_{2} f_{2}^{2}}{a_{1} \rho_{1}^{2}} l \tau, \quad \tau \in[0, T], \\
\|u(\tau, \cdot)\|_{L_{\infty}([0, l])} & \leqslant \frac{\left(3 \rho_{2}\right)^{1 / 2} f_{2}}{a_{1}^{1 / 2} \rho_{1}} l \tau^{1 / 2}, \quad \tau \in[0, T], \\
\int_{0}^{T}\|u(\tau, \cdot)\|_{L_{\infty}([0, l])} d \tau & \leqslant \frac{2\left(3 \rho_{2}\right)^{1 / 2} f_{2}}{3 a_{1}^{1 / 2} \rho_{1}} l T^{3 / 2}, \\
\left\|u_{x x}\right\|_{L_{2}\left(Q_{T}\right)}^{2} & \leqslant \frac{3 \rho_{2}^{2} f_{2}^{2}}{a_{1}^{2} \rho_{1}^{2}}\left[\left(K_{b}^{2}+\frac{K_{d}^{2} l^{2}}{2}\right) \frac{3 \rho_{2} T}{2 a_{1} \rho_{1}^{2}}+1\right] l T .
\end{aligned}
$$


ДокАЗАТЕЛЬСтво. Перепишем уравнение (1.1) в виде

$$
-u_{t}+\frac{a(x)}{\rho(t, x)} u_{x x}-\frac{b(x)}{\rho(t, x)} u_{x}-\frac{d(t, x)}{\rho(t, x)} u=-\frac{f(t, x)}{\rho(t, x)} .
$$

Умножим (2.12) на $u_{x x}$ и проинтегрируем по $Q_{\tau}, \tau \in[0, T]$. Учитывая (1.2), (1.3), (А)-(C) и применяя неравенство (1.6), после несложных преобразований приходим к неравенству

$$
\begin{aligned}
& \frac{1}{2}\left\|u_{x}(\tau, \cdot)\right\|_{L_{2}([0, l])}^{2}+\frac{a_{1}}{\rho_{2}}\left\|u_{x x}\right\|_{L_{2}\left(Q_{\tau}\right)}^{2} \\
& \quad \leqslant \frac{3 \varepsilon}{2}\left\|u_{x x}\right\|_{L_{2}\left(Q_{\tau}\right)}^{2}+\frac{1}{2 \varepsilon} \frac{K_{b}^{2}}{\rho_{1}^{2}}\left\|u_{x}\right\|_{L_{2}\left(Q_{\tau}\right)}^{2}+\frac{1}{2 \varepsilon} \frac{K_{d}^{2}}{\rho_{1}^{2}}\|u\|_{L_{2}\left(Q_{\tau}\right)}^{2}+\frac{1}{2 \varepsilon} \frac{1}{\rho_{1}^{2}}\|f\|_{L_{2}\left(Q_{\tau}\right)}^{2} .
\end{aligned}
$$

Применяя неравенство Пуанкаре-Стеклова (1.7) для оценки третьего слагаемого в правой части (2.13), получим

$$
\begin{aligned}
& \frac{1}{2}\left\|u_{x}(\tau, \cdot)\right\|_{L_{2}([0, l])}^{2}+\frac{a_{1}}{\rho_{2}}\left\|u_{x x}\right\|_{L_{2}\left(Q_{\tau}\right)}^{2} \\
& \quad \leqslant \frac{3 \varepsilon}{2}\left\|u_{x x}\right\|_{L_{2}\left(Q_{\tau}\right)}^{2}+\frac{1}{2 \varepsilon} \frac{1}{\rho_{1}^{2}}\left(K_{b}^{2}+\frac{K_{d}^{2} l^{2}}{2}\right)\left\|u_{x}\right\|_{L_{2}\left(Q_{\tau}\right)}^{2}+\frac{1}{2 \varepsilon} \frac{1}{\rho_{1}^{2}}\|f\|_{L_{2}\left(Q_{\tau}\right)}^{2} .
\end{aligned}
$$

Положим в $(2.14) \varepsilon=a_{1} / 3 \rho_{2}$. Тогда получим оценку

$$
\begin{aligned}
& \frac{1}{2}\left\|u_{x}(\tau, \cdot)\right\|_{L_{2}([0, l])}^{2}+\frac{a_{1}}{2 \rho_{2}}\left\|u_{x x}\right\|_{L_{2}\left(Q_{\tau}\right)}^{2} \\
& \quad \leqslant \frac{3 \rho_{2}}{2 a_{1} \rho_{1}^{2}}\left(K_{b}^{2}+\frac{K_{d}^{2} l^{2}}{2}\right)\left\|u_{x}\right\|_{L_{2}\left(Q_{\tau}\right)}^{2}+\frac{3 \rho_{2}}{2 a_{1} \rho_{1}^{2}}\|f\|_{L_{2}\left(Q_{\tau}\right)}^{2} .
\end{aligned}
$$

Применив неравенство (1.8) для оценки снизу второго слагаемого в левой части (2.15), получим

$$
\left\|u_{x}(\tau, \cdot)\right\|_{L_{2}([0, l])}^{2}+\left[\frac{2 a_{1}}{\rho_{2} l^{2}}-\frac{3 \rho_{2}}{a_{1} \rho_{1}^{2}}\left(K_{b}^{2}+\frac{K_{d}^{2} l^{2}}{2}\right)\right]\left\|u_{x}\right\|_{L_{2}\left(Q_{\tau}\right)}^{2} \leqslant \frac{3 \rho_{2}}{a_{1} \rho_{1}^{2}}\|f\|_{L_{2}\left(Q_{\tau}\right)}^{2} .
$$

В силу (2.6) и (2.7) квадратная скобка в левой части этого неравенства положительна, поэтому получаем

$$
\left\|u_{x}(\tau, \cdot)\right\|_{L_{2}([0, l])}^{2} \leqslant \frac{3 \rho_{2}}{a_{1} \rho_{1}^{2}}\|f\|_{L_{2}\left(Q_{\tau}\right)}^{2}
$$

Из оценки (2.16) с учетом условия (D) вытекает (2.8).

Неравенство (2.9) есть прямое следствие (2.8) и (1.9). Проинтегрировав неравенство (2.9) по $\tau$ в пределах от 0 до $T$, получим (2.10).

Далее, проинтегрировав неравенство (2.8) по $\tau$ в пределах от 0 до $T$, получим

$$
\left\|u_{x}\right\|_{L_{2}\left(Q_{T}\right)}^{2} \leqslant \frac{3 \rho_{2}}{2 a_{1} \rho_{1}^{2}} f_{2}^{2} l T^{2} .
$$


Поэтому из (2.15) с учетом (2.17) и (D) вытекает, что

$$
\begin{aligned}
\frac{a_{1}}{\rho_{2}}\left\|u_{x x}\right\|_{L_{2}\left(Q_{T}\right)}^{2} & \leqslant \frac{3 \rho_{2}}{a_{1} \rho_{1}^{2}}\left[\left(K_{b}^{2}+\frac{K_{d}^{2} l^{2}}{2}\right)\left\|u_{x}\right\|_{L_{2}\left(Q_{T}\right)}^{2}+f_{2}^{2} l T\right] \\
& \leqslant \frac{3 \rho_{2}}{a_{1} \rho_{1}^{2}}\left[\left(K_{b}^{2}+\frac{K_{d}^{2} l^{2}}{2}\right) \frac{3 \rho_{2}}{2 a_{1} \rho_{1}^{2}} f_{2}^{2} l T^{2}+f_{2}^{2} l T\right] \\
& =\frac{3 \rho_{2} f_{2}^{2}}{a_{1} \rho_{1}^{2}}\left[\left(K_{b}^{2}+\frac{K_{d}^{2} l^{2}}{2}\right) \frac{3 \rho_{2} T}{2 a_{1} \rho_{1}^{2}}+1\right] l T,
\end{aligned}
$$

а следовательно, верна оценка (2.11). Лемма 2.2 доказана.

ЗАмЕЧАНИЕ 2.1. Из условий (D), (1.2), (1.3) на основании принципа максимума (см., например, [8]) вытекает, что

$$
u(t, x) \geqslant 0, \quad(t, x) \in Q_{T} .
$$

Лемма 2.3. Пусть выполненъ условия (A)-(F) и (2.6). Тогда оператор B, определенный в (2.2), непрерывен как оператор из $M_{a_{1}}$ в $L_{\infty}([0, l])$, где $a_{1}$ из (2.7).

ДоказАтельство. Пусть $a^{(1)}(x), a^{(2)}(x) \in M_{a_{1}}$. Пусть $u^{(1)}(t, x), u^{(2)}(t, x)$ - обобщенные решения задачи (1.1)-(1.3) с коэффициентами $a^{(1)}(x)$ и $a^{(2)}(x)$ в уравнении (1.1) соответственно.

Положим $v(t, x)=u^{(1)}(t, x)-u^{(2)}(t, x), \widehat{a}(x)=a^{(1)}(x)-a^{(2)}(x)$. Тогда $v(t, x)$ удовлетворяет в $Q_{T}$ уравнению

$$
v_{t}-\frac{a^{(1)}(x)}{\rho(t, x)} v_{x x}+\frac{b(x)}{\rho(t, x)} v_{x}+\frac{d(t, x)}{\rho(t, x)} v=\frac{\widehat{a}(x)}{\rho(t, x)} u_{x x}^{(2)} .
$$

Повторяя рассуждения, проведенные в лемме 2.2 при выводе неравенства (2.16), приходим к оценке

$$
\left\|v_{x}(\tau, \cdot)\right\|_{L_{2}([0, l])}^{2} \leqslant \frac{3 \rho_{2}}{a_{1} \rho_{1}^{2}}\|\widehat{a}\|_{L_{\infty}([0, l])}^{2}\left\|u_{x x}^{(2)}\right\|_{L_{2}\left(Q_{\tau}\right)}^{2}, \quad \tau \in[0, T] .
$$

Учитывая доказанную в лемме 2.2 оценку (2.11), имеем отсюда, что

$$
\sup _{0 \leqslant \tau \leqslant T}\left\|v_{x}(\tau, \cdot)\right\|_{L_{2}([0, l])} \rightarrow 0 \quad \text { при } \quad\|\widehat{a}\|_{L_{\infty}([0, l])} \rightarrow 0 .
$$

Но тогда из определения оператора $B$ с учетом неравенств (1.9), (1.10) вытекает, что $\left\|B\left(a^{(1)}\right)-B\left(a^{(2)}\right)\right\|_{L_{\infty}([0, l])} \rightarrow 0$ при $\left\|a^{(1)}-a^{(2)}\right\|_{L_{\infty}([0, l])} \rightarrow 0$.

Лемма 2.3 доказана.

ЛЕмма 2.4. Пусть выполнены условия (A)-(F) и (2.6). Предположим дополнительно, что

$$
\left(F_{1}(T)-K_{b} \varphi^{*}\right)^{3 / 2} \geqslant \frac{9\left(\rho_{2} \varphi_{2} T\right)^{1 / 2} f_{2} l}{2 \rho_{1}}\left[\rho_{2} \chi(T)+\frac{2}{3}\left(\rho_{2} \chi^{*}+\rho^{*} \chi_{2}+d_{2} \chi_{2}\right) T\right] .
$$

Тогда для любой $a(x) \in M_{a_{1}}\left(\right.$ где $a_{1}$ из $\left.(2.7)\right)$

$$
B(a) \geqslant a_{1}
$$


ДокАЗАТЕЛЬство. В силу определения оператора $B$ неравенство (2.20) может быть записано в виде

$$
\begin{aligned}
F(x, T)-b(x) \varphi^{\prime}(x) \geqslant & \left(-\varphi^{\prime \prime}(x)\right) a_{1}+\rho(T, x) \chi(T) u(T, x) \\
& -\int_{0}^{T} \rho \chi_{t} u d t-\int_{0}^{T} \rho_{t} \chi u d t+\int_{0}^{T} d \chi u d t .
\end{aligned}
$$

В силу (А)-(F), (2.9), (2.10), (2.18) неравенство (2.21) будет выполнено, коль скоро выполнено неравенство

$$
F_{1}(T)-K_{b} \varphi^{*} \geqslant \varphi_{2} a_{1}+\rho_{2} \chi(T) \frac{\left(3 \rho_{2} T\right)^{1 / 2} f_{2} l}{a_{1}^{1 / 2} \rho_{1}}+\left(\rho_{2} \chi^{*}+\rho^{*} \chi_{2}+d_{2} \chi_{2}\right) \frac{2\left(3 \rho_{2} T\right)^{1 / 2} f_{2} l}{3 a_{1}^{1 / 2} \rho_{1}} T
$$

которое, очевидно, можно переписать в виде

$$
F_{1}(T)-K_{b} \varphi^{*} \geqslant \varphi_{2} a_{1}+\frac{\left(3 \rho_{2} T\right)^{1 / 2} f_{2} l}{a_{1}^{1 / 2} \rho_{1}}\left[\rho_{2} \chi(T)+\frac{2}{3}\left(\rho_{2} \chi^{*}+\rho^{*} \chi_{2}+d_{2} \chi_{2}\right) T\right] .
$$

Нетрудно проверить, что при $a_{1}$ из (2.7) неравенство (2.22) совпадает с (2.19).

Лемма 2.4 доказана.

ЛЕмма 2.5. Пусть выполнены условия (A)-(F) и (2.6). Пусть а определено в (2.7). Тогда $\forall a(x) \in M_{a_{1}}$

$$
B(a) \leqslant a_{2} \equiv 2\left(\rho_{2} \chi^{* *}+\rho^{* *} \chi_{2}+d_{1} \chi_{2}\right) \frac{\left(\rho_{2} \varphi_{2}\right)^{1 / 2} T^{3 / 2} f_{2} l}{\rho_{1} \varphi_{1}\left(F_{1}(T)-K_{b} \varphi^{*}\right)^{1 / 2}}+\frac{F_{2}(T)+K_{b} \varphi^{*}}{\varphi_{1}} .
$$

ДокАЗАТЕЛЬСтво. В силу определения оператора $B$, условий $(\mathrm{A})-(\mathrm{F})$ и оценок (2.9), (2.10), (2.18) имеем

$$
B(a) \leqslant \frac{2 \sqrt{3}}{3 \varphi_{1}}\left(\rho_{2} \chi^{* *}+\rho^{* *} \chi_{2}+d_{1} \chi_{2}\right) \frac{\rho_{2}^{1 / 2} T^{3 / 2} f_{2} l}{a_{1}^{1 / 2} \rho_{1}}+\frac{F_{2}(T)+K_{b} \varphi^{*}}{\varphi_{1}} .
$$

Подставляя в это неравенство $a_{1}$ из (2.7), получаем оценку (2.23). Лемма 2.5 доказана.

Обозначим теперь $M_{a_{1}}^{a_{2}}=\left\{a(x) \in L_{\infty}([0, l]): a_{1} \leqslant a(x) \leqslant a_{2}\right\}$, где $a_{1}$ из $(2.7)$, а $a_{2}$ из (2.23).

ЛЕмма 2.6. Пусть выполнены условия (A)-(F) и (2.6). Пусть а определено в (2.7), $a_{2}-$ в (2.23). Тогда оператор $B$ является вполне непрерывным оператором

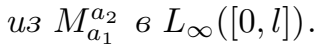

ДокАЗАтеЛЬство. Пусть $a(x) \in M_{a_{1}}^{a_{2}}$. Пусть $u(t, x)$ - обобщенное решение прямой задачи (1.1)-(1.3) с выбранной $a(x)$ в уравнении (1.1). Тогда в силу [7] $u(t, x) \in$ $C^{1, \gamma}\left(Q_{T}\right)$, причем

$$
|u|_{C^{1, \gamma}\left(Q_{T}\right)} \leqslant c,
$$

где $c=$ const $>0$ зависит от $l, T, \rho_{1}, \rho_{2}, f_{1}, f_{2}, K_{b}, k_{d}, a_{1}, a_{2}$. 
В силу оценки (2.24), компактности вложения пространства $C^{1, \gamma}\left(Q_{T}\right)$ в пространство $C\left(Q_{T}\right)$ и определения оператора $B$ следует, что оператор $B$ является компактным оператором из $M_{a_{1}}^{a_{2}}$ в $L_{\infty}([0, l])$.

В силу леммы 2.3 данный оператор непрерывен как оператор из $M_{a_{1}}^{a_{2}}$ в $L_{\infty}([0, l])$. Следовательно, оператор $B$ является вполне непрерывным оператором из $M_{a_{1}}^{a_{2}}$ в пространстве $L_{\infty}([0, l])$. Лемма 2.6 доказана.

ТеОрема 2.1. Пусть выполнены условия (A)-(F), (2.6), (2.19). Тогда существует обобщенное решение $\{u(t, x), a(x)\}$ обратной задачи (1.1)-(1.4), причем

$$
a_{1} \leqslant a(x) \leqslant a_{2},
$$

где $a_{1}$ из (2.7), а аз из (2.23), а для функции и(t,x) справедливы оценки (2.8)-(2.11), $(2.18),(2.24)$.

ДокАЗАТЕЛЬство. В силу лемм 2.4-2.6 оператор $B$ является вполне непрерывным оператором, переводящим выпуклое замкнутое множество $M_{a_{1}}^{a_{2}}$ из пространства $L_{\infty}([0, l])$ в себя. Поэтому по теореме Шаудера о неподвижной точке (см. [9; с. 193]) существует решение $a(x)$ уравнения (2.3), удовлетворяющее оценке $(2.25)$.

Но тогда в силу леммы 2.1 существует обобщенное решение $\{u(t, x), a(x)\}$ задачи (1.1)-(1.4), причем в силу леммы 2.2 справедливы оценки (2.8)-(2.11). Кроме того, в силу замечания 2.1 и леммы 2.6 выполнены также оценки (2.18) и (2.24).

Теорема доказана.

\section{3. Единственность решения обратной задачи (1.1)-(1.4).}

Теорема 3.1. Пусть выполнены условия (A)-(F), (2.6). Пусть константа $a_{1}$ определена в (2.7). Предположим дополнительно, что справедливо неравенство

$$
\frac{\rho_{2}^{3 / 2} f_{2} l T^{1 / 2}}{\varphi_{1} a_{1}^{3 / 2} \rho_{1}^{2}}\left[\left(K_{b}^{2}+\frac{K_{d}^{2} l^{2}}{2}\right) \frac{3 \rho_{2} T}{2 a_{1} \rho_{1}^{2}}+1\right]^{1 / 2}\left[3 \rho_{2} \chi(T)+2 T\left(\rho_{2} K_{\chi}+K_{\rho} \chi_{2}+K_{d} \chi_{2}\right)\right]<1 .
$$

Тогда не существует двух различных обобщенных решений $\left\{u^{(i)}(t, x), a^{(i)}(x)\right\}$, $i=1,2$, обратной задачи (1.1)-(1.4), для которьх

$$
a^{(i)}(x) \geqslant a_{1}, \quad i=1,2 .
$$

ДокАЗАТЕЛЬСтво. Предположим от противного, что существуют два различных обобщенных решения $\left\{u^{(1)}(t, x), a^{(1)}(x)\right\}$ и $\left\{u^{(2)}(t, x), a^{(2)}(x)\right\}$ задачи (1.1)-(1.4), для которых выполнено условие (3.2). Тогда обязательно

$$
a^{(1)}(x) \not \equiv a^{(2)}(x), \quad x \in[0, l],
$$

иначе $u^{(1)}(t, x) \equiv u^{(2)}(t, x)$ в $Q_{T}$ в силу единственности обобщенного решения прямой задачи (1.1)-(1.3) (см. [7]).

Положим $v(t, x)=u^{(1)}(t, x)-u^{(2)}(t, x), \widehat{a}(x)=a^{(1)}(x)-a^{(2)}(x)$. Тогда пара $\{v(t, x)$, $\widehat{a}(x)\}$ удовлетворяет соотношениям

$$
\begin{gathered}
\rho(t, x) v_{t}-a^{(1)}(x) v_{x x}+b(x) v_{x}+d(t, x) v=\widehat{a}(x) u_{x x}^{(2)}, \quad(t, x) \in Q_{T}, \\
v(0, x)=0, \quad x \in[0, l], \\
v(t, 0)=v(t, l)=0, \quad t \in[0, T], \\
\int_{0}^{T} v(t, x) \chi(t) d t=0, \quad x \in[0, l] .
\end{gathered}
$$


Повторяя доказательство леммы 2.2 и учитывая доказанную оценку (2.11), получаем оценки

$$
\begin{gathered}
\|v(T, \cdot)\|_{L_{\infty}([0, l])} \leqslant \frac{3 \rho_{2}^{3 / 2} f_{2} l T^{1 / 2}}{a_{1}^{3 / 2} \rho_{1}^{2}}\left[\left(K_{b}^{2}+\frac{K_{d}^{2} l^{2}}{2}\right) \frac{3 \rho_{2} T}{2 a_{1} \rho_{1}^{2}}+1\right]^{1 / 2}\|\widehat{a}\|_{L_{\infty}([0, l])}, \\
\int_{0}^{T}\|v(t, \cdot)\|_{L_{\infty}([0, l])} d t \leqslant \frac{2 \rho_{2}^{3 / 2} f_{2} l T^{3 / 2}}{a_{1}^{3 / 2} \rho_{1}^{2}}\left[\left(K_{b}^{2}+\frac{K_{d}^{2} l^{2}}{2}\right) \frac{3 \rho_{2} T}{2 a_{1} \rho_{1}^{2}}+1\right]^{1 / 2}\|\widehat{a}\|_{L_{\infty}([0, l])} .
\end{gathered}
$$

Умножим теперь соотношение (3.4) на $\chi(t)$ и проинтегрируем по $t$ в пределах от 0 до $T$. Учитывая (3.5)-(3.7), получим после интегрирования по частям

$$
\begin{aligned}
\widehat{a}(x)= & \frac{1}{\varphi^{\prime \prime}(x)} \rho(T, x) \chi(T) v(T, x) \\
& \quad+\frac{1}{\varphi^{\prime \prime}(x)} \int_{0}^{T}\left[d(t, x) \chi(t)-(\rho(t, x) \chi(t))_{t}\right] v(t, x) d t .
\end{aligned}
$$

Обозначим правую часть соотношения (3.10) через $J(x)$ и оценим ее в норме пространства $L_{\infty}([0, l])$, используя условия $(\mathrm{A})-(\mathrm{F})$, оценки $(3.8),(3.9)$ и условие $(3.1)$. Имеем

$$
\begin{aligned}
\|J(x)\|_{L_{\infty}([0, l]) \leqslant} \leqslant & \frac{\rho_{2} \chi(T)}{\varphi_{1}}\|v(T, \cdot)\|_{L_{\infty}([0, l])} \\
& \quad+\frac{1}{\varphi_{1}}\left(\rho_{2} K_{\chi}+K_{\rho} \chi_{2}+K_{d} \chi_{2}\right) \int_{0}^{T}\|v(t, \cdot)\|_{L_{\infty}([0, l])} d t \\
\leqslant & \frac{\rho_{2}^{3 / 2} f_{2} l T^{1 / 2}}{\varphi_{1} a_{1}^{3 / 2} \rho_{1}^{2}}\left[\left(K_{b}^{2}+\frac{K_{d}^{2} l^{2}}{2}\right) \frac{3 \rho_{2} T}{2 a_{1} \rho_{1}^{2}}+1\right]^{1 / 2} \\
& \times\left[3 \rho_{2} \chi(T)+2 T\left(\rho_{2} K_{\chi}+K_{\rho} \chi_{2}+K_{d} \chi_{2}\right)\right]\|\widehat{a}\|_{L_{\infty}([0, l])} \\
< & \|\widehat{a}\|_{L_{\infty}([0, l])} .
\end{aligned}
$$

Из соотношений (3.10) и (3.11), очевидно, следует, что $\|\widehat{a}\|_{L_{\infty}([0, l])}=0$, т.е., что $a^{(1)}(x)=a^{(2)}(x)$ почти всюду на отрезке [0,l], а это противоречит (3.3).

Теорема 3.1 доказана.

4. Примеры выполнения условий теорем существования и единственности решения задачи (1.1)-(1.4). Исследуем вопрос о выполнении дополнительных условий (2.6), (2.19) и (3.1), наложенных в теоремах 2.1 и 3.1.

ПримеР 4.1. Рассмотрим в $Q_{T}$ обратную задачу для уравнения

$$
\rho(t, x) u_{t}-a(x) u_{x x}=1
$$

с начальным и граничными условиями (1.2), (1.3) и условием переопределения

$$
\int_{0}^{T} u(t, x) t(T-t) d t=x(l-x), \quad x \in[0, l],
$$

где $\rho(t, x)$ - измеримая функция, удовлетворяющая условию (A). 
Для этой задачи имеем

$$
\begin{gathered}
K_{b}=K_{d}=0, \quad \chi_{1}=0, \quad \chi_{2}=\frac{T^{2}}{4}, \quad \chi^{*}=\chi^{* *}=T, \quad \varphi^{\prime \prime}=-2, \\
a_{1}=\frac{T^{3}}{36}, \quad F_{1}(T)=\frac{T^{3}}{6} .
\end{gathered}
$$

Условие (2.6), очевидно, выполнено. Условие (2.19) имеет вид

$$
36 \sqrt{3} \frac{\rho_{2}^{1 / 2}}{\rho_{1}}\left(\rho_{2}+\rho^{*} \frac{T}{4}\right) \frac{l}{T^{2}} \leqslant 1,
$$

а условие (3.1) - вид

$$
216 \frac{\rho_{2}^{3 / 2}}{\rho_{1}^{2}}\left(\rho_{2}+K_{\rho} \frac{T}{4}\right) \frac{l}{T^{2}}<1 .
$$

Очевидно, оба условия (4.3), (4.4) выполняются, если либо $l$ достаточно мало, либо $T$ достаточно велико.

При выполнении указанных условий справедливы теоремы существования и единственности решения обратной задачи (4.1), (1.2), (1.3), (4.2).

ПримеР 4.2. Рассмотрим в $Q_{T}$ обратную задачу для уравнения

$$
\left(2+\sin \frac{x t}{T}\right) u_{t}-a(x) u_{x x}=1
$$

с начальным и граничными условиями (1.2), (1.3) и условием переопределения

$$
\frac{1}{T} \int_{0}^{T} u(t, x) d t=x(l-x), \quad x \in[0, l] .
$$

Заметим, что условие переопределения (4.6) имеет простой физический смысл: это взятие среднего по времени от функции $u(t, x)$.

Для задачи (4.5), (1.2), (1.3), (4.6) имеем

$$
\begin{gathered}
\rho_{1}=1, \quad \rho_{2}=3, \quad \rho^{*}=\rho^{* *}=\frac{l}{T}, \quad K_{b}=K_{d}=0, \quad \chi_{1}=\chi_{2}=\frac{1}{T}, \\
\chi^{*}=\chi^{* *}=0, \quad \varphi^{\prime \prime}=-2, \quad F_{1}(T)=1, \quad a_{1}=\frac{1}{6} .
\end{gathered}
$$

Условие (2.6), очевидно, выполнено. Условие (2.19) запишется в виде

$$
\frac{3 \sqrt{6}}{2}(9+2 l) \frac{l}{T^{1 / 2}} \leqslant 1,
$$

а условие (3.1) в виде

$$
27 \sqrt{2}(9+2 l) \frac{l}{T^{1 / 2}}<1 .
$$

Как и в случае примера 4.1, оба условия (4.7) и (4.8) выполняются, если либо $l$ достаточно мало, либо $T$ достаточно велико.

При выполнении указанных условий также справедливы теоремы существования и единственности решения обратной задачи (4.5), (1.2), (1.3), (4.6).

Автор благодарит А. И. Прилепко, А. Б. Костина, Д. Г. Орловского и В. В. Соловьева за полезное обсуждение результатов работы. 


\section{СПИСОК ЦИТИРОВАННОЙ ЛИТЕРАТУРЫ}

[1] А.И. Прилепко, А.Б. Костин, "Об обратных задачах определения коэффициента в параболическом уравнении. I", Сиб. матем. журн., 33:3 (1992), 146-155.

[2] А. И. Кожанов, "О разрешимости обратной задачи нахождения коэффициента теплопроводности", Сиб. матем. журн., 46:5 (2005), 1053-1071.

[3] В.М. Исаков, "Об одном классе обратных задач для параболических уравнений”, Докл. АН СССР, 263:6 (1982), 1296-1299.

[4] V. Isakov, "Inverse parabolic problems with the final overdetermination", Comm. Pure Appl. Math., 44:2 (1991), 185-209.

[5] А. М. Денисов, Введение в теорию обратных задач, Изд-во МГУ, М., 1994.

[6] О. А. Ладыженская, В. А. Солонников, Н. Н. Уральцева, Линейные и квазилинейные уравнения параболического типа, Наука, М., 1967.

[7] С.Н. Кружков, "Квазилинейные параболические уравнения и системы с двумя независимыми переменными", Тр. сем. им. И.Г. Петровского, 1979, № 5, 217-272.

[8] С.Н. Кружков, Нелинейные уравнения с частными производными, ч. 1: Эллиптические и параболические уравнения второго порядка, Изд-во МГУ, М., 1969.

[9] Л.А. Люстерник, В.И. Соболев, Краткий курс функиионального анализа, Высшая школа, М., 1982.

В. Л. Камынин

Московский государственный инженерно-физический институт

E-mail: image@consultant.ru
Поступило

23.04.2007 